\title{
Hypertrophic cardiomyopathy or hypertensive heart disease?
}

\author{
Jacek Migaj, Marta Kałużna-Oleksy, Magdalena Janus, Magdalena Dudek, Maciej Lesiak, Zofia Oko-Sarnowska
}

$1^{\text {st }}$ Department of Cardiology, Poznan University of Medical Sciences, Poznań, Poland

\author{
Correspondence to: \\ Jacek Migaj, MD, PhD, \\ $1^{\text {st }}$ Department \\ of Cardiology, \\ Poznan University \\ of Medical Sciences, \\ University Hospital \\ of Lord's Transfiguration, \\ Długa $1 / 2$, \\ 61-848 Poznań, Poland, \\ phone: +48618549146, \\ e-mail: \\ jacek.migaj@skpp.edu.pl \\ Copyright by the \\ Author(s), 2021 \\ Kardiol Pol. 2021; \\ 79 (7-8): 883-884; \\ DOI: 10.33963/KP.a2021.0017 \\ Received: \\ April 20, 2021 \\ Revision accepted: \\ May 19, 2021 \\ Published online: \\ May 21, 2021
}

A 49-year-old male patient, with a history of typical angina, poorly controlled arterial hypertension, hypercholesterolemia, and smoking presented to a regional hospital because of chronic coronary syndrome exacerbation. Coronary angiography revealed a multivessel coronary artery disease with in-stent restenosis in the left circumflex artery and chronic total occlusion of the left anterior descending artery (Figure 1B-C). Based on echocardiographic findings, hypertrophic cardiomyopathy (HCM) was suspected, and the patient was disqualified from coronary artery bypass grafting (CABG). The patient was referred to our tertiary health care center for further management, including high-risk percutaneous coronary interventions.

The stent in the left circumflex artery was deployed in 2014. However, angina symptoms reappeared two years later, and the patient was treated pharmacologically for nearly four years in the outpatient setting without any further diagnostic work-up.

On admission to our Department of Cardiology, the patient had no chest pain, and electrocardiogram showed negative $T$ waves in $\mathrm{I}, \mathrm{aVL}$, and V3-V6, ST depression in I and $\mathrm{aVL}$, and ST elevation with q waves in III, aVF, and V1-V2 leads (Figure 1A). These changes were observed previously and initially interpreted as signs of a previous myocardial infarction(s) without signs of cardiac hypertrophy. Echocardiography performed on admission showed the maximum interventricular septum (IVS) thickness of $22 \mathrm{~mm}$ and the maximum left ventricle (LV) free wall thickness of $12 \mathrm{~mm}$, without evidence of LV outflow tract obstruction, with $E / E^{\prime} 13$, with global longitudinal strain $-9.2 \%$, and without impairment of LV segmental contractility. However, the years-long history of poorly controlled arterial hypertension (on 6 antihypertensive drugs) raised doubts as to the suspicion of HCM. And the differential diagnostics of these two conditions based solely on echocardiographic imaging can be confusing [1]. Therefore, cardiac magnetic resonance (CMR) was performed to provide high-quality information that can support the HCM diagnosis [2]. CMR revealed an asymmetric LV hypertrophy with the maximum IVS thickness of $24 \mathrm{~mm}$ and the maximum LV free wall thickness of $16 \mathrm{~mm}$. Additionally, we observed the presence of a mid-wall late gadolinium enhancement (LGE) in the hypertrophied mid and basal segments of the interventricular septum, i.e., a typical non-ischemic pattern of fibrosis in the course of HCM. CMR also showed an ischemic subendocardial LGE in the lateral and inferolateral walls, a characteristic of a past myocardial infarction (Figure 1D-E).

The patient reported recurring chest pain at rest during hospitalization that required nitroglycerin administration (without any new electrocardiogram changes or troponin elevation). In consequence, we changed the initial diagnosis of chronic coronary syndrome to unstable angina. Considering the advancement of coronary lesions, the patient's case was again consulted on the Heart Team meeting, and cardiac surgeons qualified the patient for an urgent $C A B G$.

An uneventful CABG was performed during the same hospital stay. The patient has successfully completed the rehabilitation and is now free of angina symptoms. Due to the low sudden cardiac death risk, the patient was not qualified for implantation of cardioverter-defibrillator [3,4]. The patient is under constant care supervised by an HCM expert in the Outpatient Clinic at our Hospital.

The discharge diagnoses were unstable angina, previous myocardial infarction, lowrisk HCM without left ventricular outflow tract obstruction, and poorly controlled hypertension. 


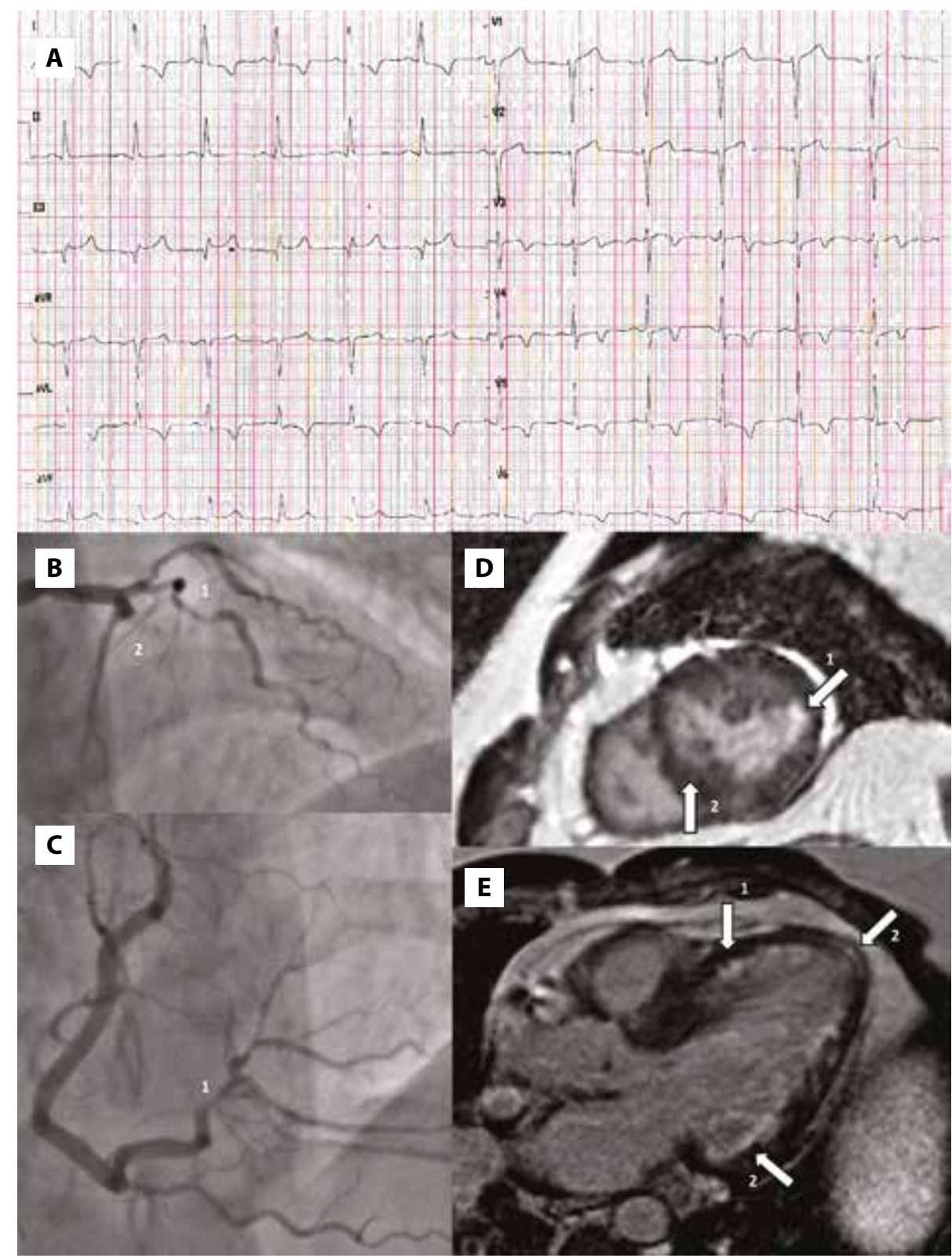

Figure 1. A. Electrocardiogram reveals likely signs of previous myocardial infarctions of the lateral and inferior walls signs of cardiac hypertrophy. B. Coronary angiography shows multiple atherosclerotic lesions with proximal occlusions of the left anterior descending artery (1) and the left circumflex artery $(2$, in-stent restenosis). C. Coronary angiography shows non-obstructive lesions in the right coronary artery with critical stenosis in the posterior descending artery (1). D. Cardiac magnetic resonance late gadolinium enhancement image, short-axis view, shows a subendocardial enhancement of the inferolateral wall characteristic of a previous myocardial infarction (1) and a non-ischemic mid-wall late gadolinium enhancement typical of fibrosis in the course of hypertrophic cardiomyopathy (2). E. Cardiac magnetic resonance late gadolinium enhancement image, three-chamber view, shows a non-ischemic midwall late gadolinium enhancement typical of fibrosis in the course of hypertrophic cardiomyopathy (1) and ischemic subendocardial late gadolinium enhancement typical of a previous myocardial infarction (2): asymmetric mid septal hypertrophy with a maximum wall thickness of $24 \mathrm{~mm}$ can be observed

\section{Article information}

Conflict of interest: None declared.

Open access: This article is available in open access under Creative Common Attribution-Non-Commercial-No Derivatives 4.0 International (CC BY-NC-ND 4.0) license, allowing to download articles and share them with others as long as they credit the authors and the publisher, but without permission to change them in any way or use them commercially. For commercial use, please contact the journal office at kardiologiapolska@ptkardio.pl.

How to cite: Migaj J, Kałużna-Oleksy M, Janus M. Hypertrophic cardiomyopathy or hypertensive heart disease? Kardiol Pol. 2021; 79(7-8): 883-884, doi: 10.33963/KP.a2021.0017.

\section{REFERENCES}

1. Gawor M, Franaszczyk M, Kowalik E, et al. A young patient with left ventricular hypertrophy and accidentally discovered aortic dissection: hypertensive heart disease or hypertrophic cardiomyopathy? Kardiol Pol. 2020; 78(2): 171-173, doi: 10.33963/kp.15159, indexed in Pubmed: 31994492.

2. Dorobantu L, Ticulescu R, Greavu M, et al. Current management and surgical advances in patients with hypertrophic obstructive cardiomyopathy. Kardiol Pol. 2019; 77(9):829-836, doi: 10.33963/KP.14965, indexed in Pubmed: 31495825.

3. Elliott PM, Anastasakis A, Borger MA, et al. 2014 ESC guidelines on diagnosis and management of hypertrophic cardiomyopathy: the task force for the diagnosis and management of hypertrophic cardiomyopathy of the European Society of Cardiology (ESC). Eur Heart J. 2014; 35(39):2733-2779, doi: 10.1093/eurheartj/ehu284, indexed in Pubmed: 25173338.

4. O'Mahony C, Jichi F, Pavlou M, et al. Hypertrophic Cardiomyopathy Outcomes Investigators. A novel clinical risk prediction model for sudden cardiac death in hypertrophic cardiomyopathy (HCM risk-SCD). Eur Heart J. 2014; 35(30): 2010-2020, doi: 10.1093/eurheartj/eht439, indexed in Pubmed: 24126876. 